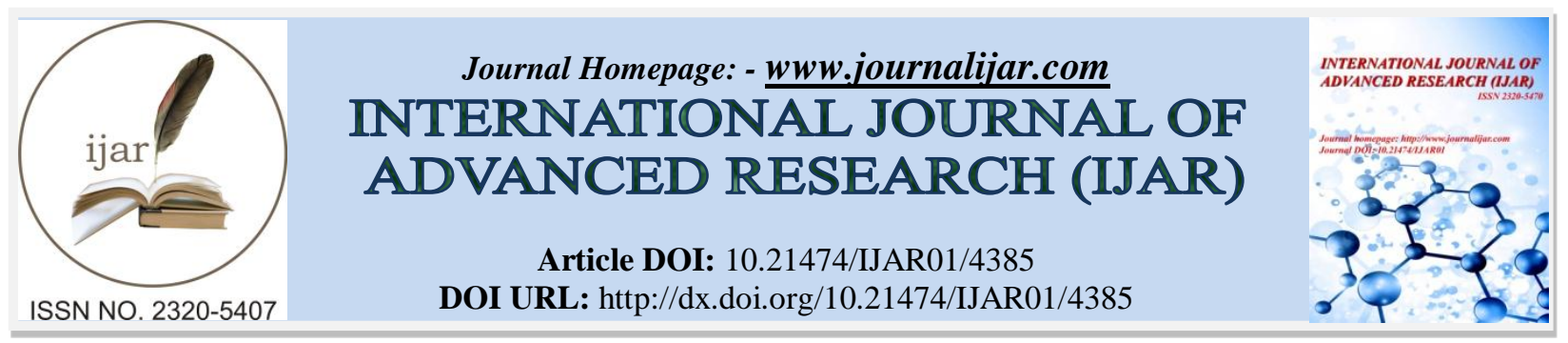

RESEARCH ARTICLE

\title{
FONCTIONNEMENT HYDRODYNAMIQUE DES BAIES DE CINTRA ET DE DAKHLA (SUD DU
} MAROC).

\author{
Hilmi Karim ${ }^{1}$, Makaoui Ahmed ${ }^{1}$, Ettahiri Omar ${ }^{1}$, Idrissi Mohammed ${ }^{1}$, Abdellaoui Benyounes ${ }^{1}$, Larissi \\ Jamila $^{1}$, El Ouehabi Zineb ${ }^{1}$ and Bessa Ismail ${ }^{2}$. \\ 1. Institut National de Recherche Halieutique Casablanca, Maroc. \\ 2. Université Hassan II Faculté des Sciences Ben M'Sik, Casablanca, Maroc.
}

\section{Manuscript Info}

\section{Manuscript History}

Received: 03 april 2017

Final Accepted: 05 june 2017

Published: june 2017

Key words:-

Cintra and Dakhla's bay- Dakhla Oued

Eddahab area - South of Morocco-

Marine circulation- 2D hydrodynamic model.

\section{Abstract}

The marine circulation of Cintra $\left(23^{\circ} 08^{\prime} \mathrm{N}-16^{\circ} 20^{\prime} \mathrm{W}\right)$ and Dakhla $\left(23^{\circ} 30^{\prime} \mathrm{N}-16^{\circ} \mathrm{W}\right)$ bays located at the Dakhla Oued Eddahab area in the south of Morocco have been studied from October to November 2016 by $2 \mathrm{D}$ hydrodynamic model. This study aims to understand their marine circulation and to compare their hydrodynamic functioning. Mainly governed by M2 tide and wind, currents in Dakhla bay are bidirectional and very strong in intensity near the inlet of the bay and its surrounding area (between 1 and $2 \mathrm{~m} \cdot \mathrm{s}^{-1}$ ) during spring tides. During the neap tides, their intensity are less than $0.6 \mathrm{~m} \cdot \mathrm{s}^{-1}$ in its central and northern parts of the bays. At Cintra's bay, the currents are generally strong in intensity (mainly $>0.5 \mathrm{~m} . \mathrm{s}^{-1}$ ) outside the bay. Inside this bay, their intensity are less than $0.3 \mathrm{~m} \cdot \mathrm{s}^{-1}$ and they are generally oriented to the south of the bay due to the influence of the trade winds ( $\mathrm{N}$ to $\mathrm{NE}$ ),

Copy Right, IJAR, 2017,. All rights reserved.

\section{Introduction:-}

Dans le domaine marin et depuis ces dernières décennies, la modélisation numérique à travers le globe a pris une avancée telle qu'elle est devenue un outil incontournable pour simuler les phénomènes naturels dans les régions côtières et littorales. Au Maroc, les études océanographiques sur les sites littoraux des façades atlantique et méditerranéenne (Figure 1) sont très variées: Abdellaoui et al., 2015; Alaoui et al., 2010; Arid et al., 2005; Bocci et al., 2016; Damsiri et al., 2015; El Khalidi et al., 2014; Guelorget et al., 1987; Koutitonsky et al., 2006, 2007, 2012; Hamoumi, 2012; Hassou et al., 2014; Hilmi et al., 2005 a, b et c, 2009; Idardare et al ., 2013; Labbardi et al., 2005 ; Lakhdar et al., 2001, 2004, 2005; Maanan et al., 2014, 2015; Mostarih et al., 2016; Najih et al., 2016; Orbi et al., 1996; Saad et al., 2013, 2015; Zidane, 2011; Zidane et al., 2008, 2013 a et b,; Zourarah et al., 2012, ... Par contre, les études portant sur la modélisation hydrodynamique des lagunes et baies du Maroc sont très peu nombreuses. On citera, par exemple pour le complexe lagunaire de Oualidia/Sidi Moussa, les travaux de Hilmi et al. (2005b); Koutistonsky et al. (2007 et 2012); Orbi et al. (2008); Flower et al. (2009) pour la lagune de Moulay Bousselham («Merja Zerga ») situées sur la façade atlantique marocaine. Pour la lagune de Nador (« Marchica») située sur la façade méditerranéenne marocaine, on citera les travaux de Hilmi et al., (2005c, 2015); Koutistonsky et al. (2006); Yachouti et al., 2014; Umgeisser et al. (2005, 2014); Bajo et al. (2017),... S'agissant des baies de Dakhla et Cintra faisant l'objet de ce travail (figure 1), les études en modélisation hydrodynamique sont quasi inexistantes, mis à part celles de Hilmi et al. (2017 a et b). L'objectif de ce travail est de décrire, à l'aide d'un modèle hydrodynamique bidimensionnel, les circulations marines des baies de Cintra et Dakhla, de comprendre les processus physiques 
régissant leur fonctionnement hydrodynamique et de comparer leur mode de fonctionnement hydrodynamique, en étant soumises aux mêmes conditions météorologiques et de marée sur la période d'étude allant d'octobre à novembre 2016.

\section{Présentation des deux sites d'étude :-}

Localisées dans la province de Dakhla Oued Eddahab (sud du Royaume du Maroc), les baies de Cintra et de Dakhla (figure 1) font partie des plus importantes baies du littoral national. Séparée de l'océan Atlantique par la péninsule de Oued Eddahab, la baie de Dakhla $\left(23^{\circ} 30^{\prime} \mathrm{N}-16^{\circ} \mathrm{W}\right)$ présente une longueur de $37 \mathrm{~km}$ environ avec une largeur variant entre 10 à $12 \mathrm{~km}$ environ. Orientée dans l'axe NNE-SSW, elle communique avec l'Océan Atlantique du côté Sud à travers une large passe de $13 \mathrm{~km}$ environ d'ouverture (figure 1a). Sous forme concave et orientée selon l'axe NW-SW, la baie de Cintra $\left(23^{\circ} 08^{\prime} \mathrm{N}-16^{\circ} 20^{\prime} \mathrm{W}\right)$ présente une longueur de $21 \mathrm{~km}$ avec une largeur de $11 \mathrm{~km}$ environ dans sa zone centrale et communique avec l'Océan Atlantique à travers sa large passe (figure 1b). Le climat de ces deux zones d'étude est présenté en détails dans Hilmi et al. (2017 a et b).

\section{Materiel and Methodes:-}

Le modèle bidimensionnel (2D), implémenté pour les baies de Dakhla et Cintra, est le modèle MIKE21-HD développé par la société DHI dont les équations du modèle 2D sont décrites en détail (DHI, 1990). Le modèle MIKE21/HD simule l'évolution temporelle des courants et du niveau d'eau,

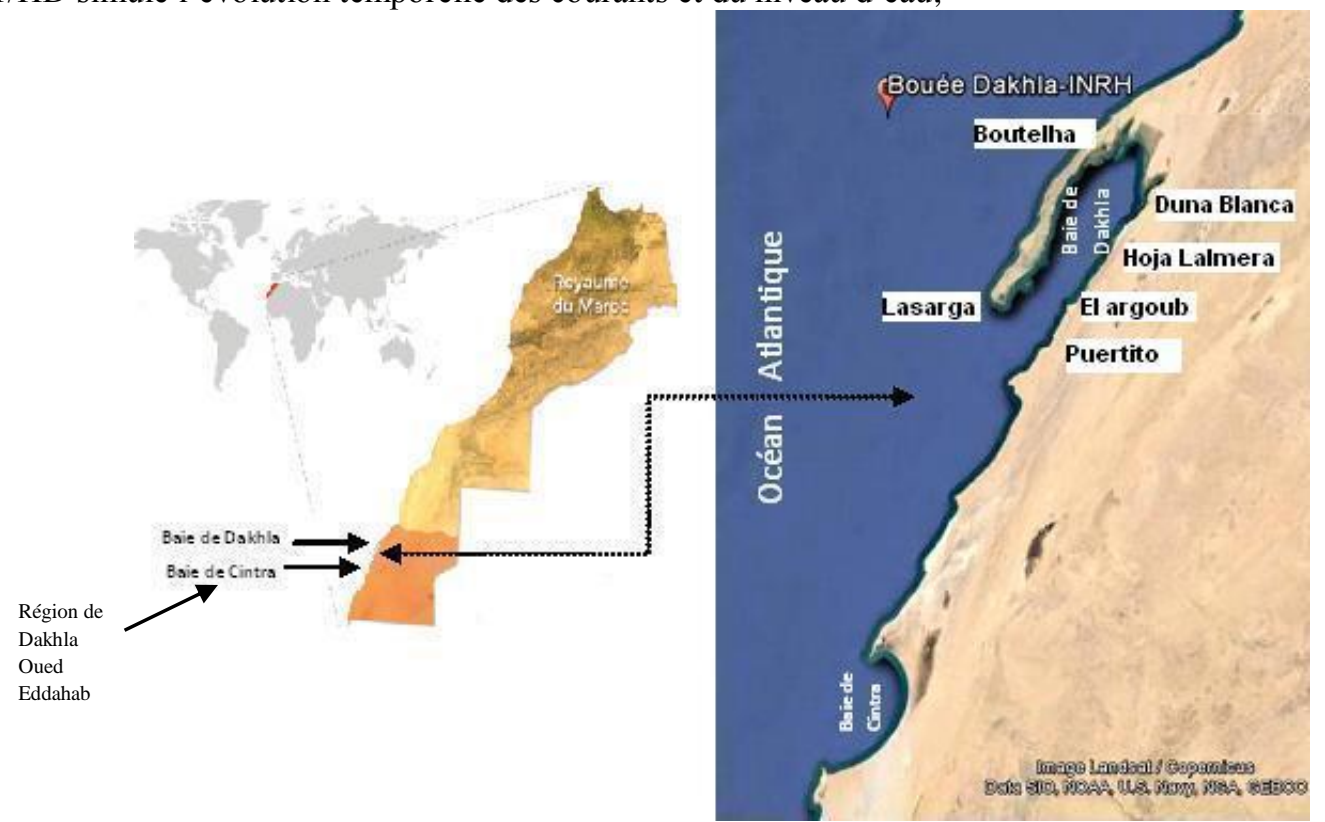

Figure 1 :- Situation géographique des baies de Dakhla et de Cintra de la région de Dakhla Oued Eddahab dans la zone Sud du Royaume du Maroc.

en intégrant les équations de la conservation du mouvement et de la masse intégrées sur l'axe vertical. Il a été calibré (frottement au fond, entrainement par le vent) et validé sur les deux baies à partir des mesures de terrain (Hilmi et al., $2017 \mathrm{a}$ et b). Dans cette étude, le modèle 2D n'intègre pas la houle, ni les températures de l'air/de l'eau et ni les précipitations. Les intrants du modèle $2 \mathrm{D}$ pour les deux baies sont les paramètres suivants: la bathymétrie, les marées et les vents observés sur la période de simulation allant du 07 octobre au 06 novembre 2016. Le choix de cette période de simulation a coïncidé avec les grandes marées d'équinoxe dans la région.

\section{Bathymétrie des baies de Cintra et de Dakhla :-}

La figure 2 présente la bathymétrie des baies de Cintra et de Dakhla corrigées par rapport à la marée (Hilmi et al., $2017 \mathrm{a}$ et b). Pour les besoins de cette étude en matière de modélisation, la grille de calcul développée pour le domaine de la baie de Dakhla comprend 160 mailles en X (vers l'Est) et 180 mailles en Y (vers le Nord), de dimensions $200 \mathrm{~m}$ x $200 \mathrm{~m}$ sur le plan horizontal, soit un domaine d'emprise s'étalant sur $32 \mathrm{~km}$ vers l'Est et sur 34 $\mathrm{km}$ vers le Nord (figure 3a). Pour la baie de Cintra, cette grille de calcul comprend 115 mailles en X (vers l'Est) et 
175 mailles en Y (vers le Nord), de dimensions 200m x 200m sur le plan horizontal, soit un domaine d'emprise s'étalant sur $23 \mathrm{~km}$ vers l'Est et sur $35 \mathrm{~km}$ vers le Nord (figure $3 \mathrm{~b}$ ).

a)

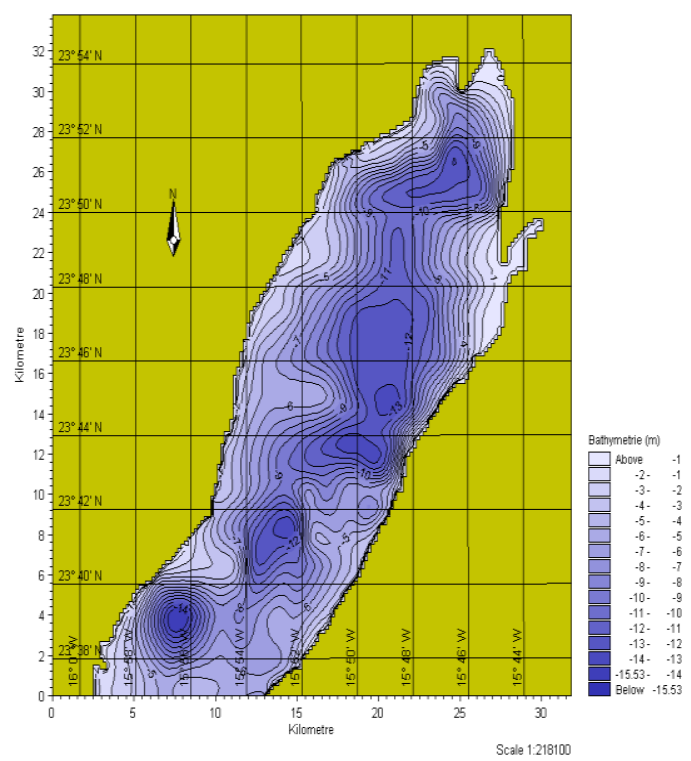

b)

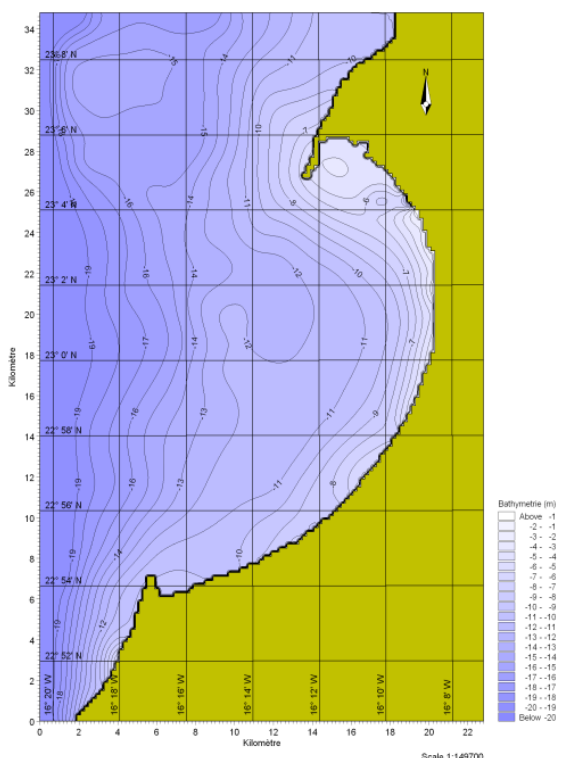

Figure 2:- Bathymétrie des baies de a) Dakhla et b) Cintra pour les domaines d'emprises du modèle2D, corrigée par rapport à la marée (Hilmi et al., 2017 a et b).

\section{Forçage par la marée :-}

Pour les besoins du modèle et aux frontières ouvertes des domaines d'emprise (Figure 3) et à défaut de marée observée dans la zone d'étude, le modèle 2D a été forcé par la marée prédite à Dakhla par le Service Hydrographique et Océanographique de la Marine Française (SHOM) sur la période du 07 octobre au 06 novembre 2016. Cette période couvre ainsi deux cycles de marée compris entre des marées de morte-eau (coefficient de marée 31) et de vive-eau (coefficient 116) coïncidant avec les grandes marées d'équinoxe (Figure 3).

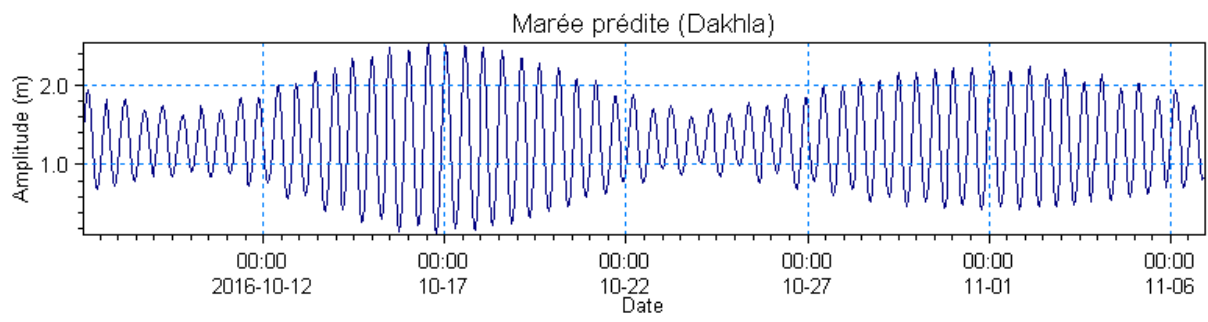

Figure 3:- Marée horaire prédite à Dakhla sur la période du 07 octobre au 06 novembre 2016 (Source données SHOM).

\section{Forçage par le vent:-}

La figure 4 présente la rose du vent horaire observé à la bouée METOCEAN «Dakhla » de l'Institut National de Recherche Halieutique $\left(23.91711^{\circ} \mathrm{N} ; \quad-16.18703^{\circ} \mathrm{W}\right)$ sur la période allant du 07 octobre au 06 novembre 2016. On constate que les vents sont de secteurs variables sur cette période de l'étude, avec des vents dominants de secteurs Nord à Nord Est (fréquence > 50\%) dont les intensités sont supérieures à $5 \mathrm{~m} . \mathrm{s}^{-1}$. Ce sont ces vents dominants qui sont généralement observés dans la zone d'étude en cette période de l'année (Hilmi et al., 2017 a et b). Les vents soufflant dans les secteurs Est, Ouest, Sud à Sud Est sont aussi observés mais avec une fréquence moins importante et leurs intensités sont inférieures à $5 \mathrm{~m}$. $\mathrm{s}^{-1}$ (Figure 4). 


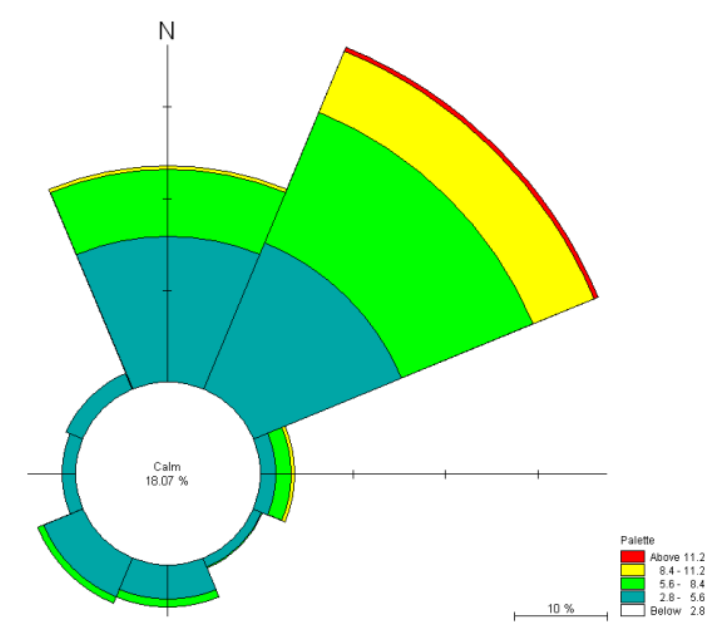

Figure 4 :- Rose du vent horaire observé à la bouée METOCEAN «Dakhla» de l'Institut Nationale de Recherche Halieutique $\left(23.91711^{\circ} \mathrm{N} ;-16.18703^{\circ} \mathrm{W}\right.$ Ref figure 1) sur la période allant du 07 octobre au 06 novembre 2016 (Hilmi et al, 2017b).

\section{Resultats et and-Discussion:-}

Les simulations de la circulation marine des baies de Cintra et de Dakhla ont porté sur la période du 07 octobre au 06 novembre 2016 couvrant deux cycles de marées de morte-eau et de vive-eau. Compte tenu de la longue période de simulation, nous présentons ici comme résultats des exemples de configuration de la circulation des deux baies selon les situations suivantes:

$>$ en période de marées de vive-eau à marées entrante et sortante: cas du 16 octobre 2016 par exemple (coefficient de marée 116) (figures 5 et 6);

$>$ en période de marées de morte-eau à marées entrante et sortante: cas du 23 octobre 2016 (coefficient de marée 31) (figures 7 et 8 ).

\section{Cas de la baie de Dakhla:-}

Quelque soit le type de marées de vive-eau et de morte-eau, les courants de marée sont intenses dans la baie de Dakhla et bidirectionnels dans l'axe Nord Est - Sud Ouest, en suivant les cycles de la marée semi diurne (de type M2). En périodes de marées de vives-eaux (coefficients de marée 116) et durant les flots, on constate que les plus forts courants se manifestent généralement en aval de la baie entre les latitudes $23^{\circ} 38^{\prime} \mathrm{N}$ et $23^{\circ} 44^{\prime} \mathrm{N}$ (soit entre Lasarga et Dakhla sur la rive Ouest de la baie) (figures 5a et 6a). Sur la rive Est de la baie, les plus forts courants dont les intensités sont comprises entre 1 et $2 \mathrm{~m} \cdot \mathrm{s}^{-1}$ se manifestent principalement entre Puertito, El Argoub et Hoja Lalmera et El Argoub. Vers l'amont de la baie et au delà des latitudes supérieures à $23^{\circ} 44^{\prime} \mathrm{N}$ (soit entre Dakhla, Boutalha et en allant vers les parcs aquacoles), les intensités du courant généralement comprises entre 0.1 et $0.5 \mathrm{~m} . \mathrm{s}^{-}$ ${ }^{1}$ (figures 5a et 6a). Durant les jusants et dans la zone centrale et vers l'amont de la baie, des cellules gyratoires cycloniques vers la rive Ouest et/ou anticycloniques vers la rive Est du courant sont observées, suite à une bathymétrie non régulière et peu profonde et à une présence d'une multitude de chenaux dans ces zones (figure 2). En périodes de marées de mortes-eaux (coefficients de marée 31), les courants de marée sont relativement plus faibles dont les intensités sont inférieures à $0.5 \mathrm{~m} . \mathrm{s}^{-1}$ dans la baie (figures $7 \mathrm{a}$ et $8 \mathrm{a}$ ). On constate que les plus forts courants se manifestent à l'entrée de la baie, entre La Sarga (coté Ouest de la baie), Argoub et Puertito (côté Est de la baie). Durant les jusants, on observe également ces cellules gyratoires du courant dans la zone centrale et vers l'amont de la bai. Bien que faibles et dû à l'influence des vents, ces cellules vont générer près des côtes des courants de dérive. Une autre particularité de la circulation marine de la baie de Dakhla est que les courants de marée sortants (jusants) sont généralement légèrement plus intenses que les courants entrants (flots), que ce soit en marées de vives eaux ou de mortes eaux (Figures 5a à 8a).

\section{Cas de la baie de Cintra:-}

En période de marée de vive eau en prenant l'exemple de la journée du 23 octobre 2016 à $13 \mathrm{~h}$ à marée entrante coïncidant avec des vents de secteur Nord Est, les courants sont orientés du Nord au Sud avec des intensités comprises entre 0.1 et $0.5 \mathrm{~m} . \mathrm{s}^{-1}$ (figure $5 \mathrm{~b}$ ). Les plus forts courants sont observés au large et vers le Nord de la baie de Cintra. A l'intérieur de cette baie, les courants sont dirigés du Nord au Sud mais avec des intensités de moindre 
amplitude (< à $0.3 \mathrm{~m} \cdot \mathrm{s}^{-1}$ ) (figure 5b). A marée sortante et en prenant l'exemple de la journée du 16 novembre 2016 à $19 \mathrm{~h}$ en période de marée de vive eau coïncidant avec des vents de secteur Nord, les courants au large de la baie ont tendance à être orientés vers le Nord Ouest avec des intensités comprises entre 0.1 et $0.3 \mathrm{~m} . \mathrm{s}^{-1}$ (figure 6b). A l'intérieur de la baie, les intensités du courant sont inférieures à $0.3 \mathrm{~m} \cdot \mathrm{s}^{-1}$. En périodes de marées morte eau, que ce soit à marées entrante ou sortante, les courants dans la baie de Cintra présentent généralement de faibles intensités (<

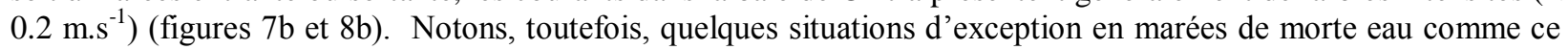
fût le cas par exemple de la journée du 09 octobre 2016 à 19h (figure 9). En effet, cette journée à coïncidé avec une période de marée de morte eau de coefficient 41 mais par très forts vents de secteur Nord Est d'intensités supérieures à $8 \mathrm{~m} \cdot \mathrm{s}^{-1}$. Les courants de surface observés au large des deux baies sont orientés dans la direction Nord Est (figure 9b). Ces courants sont de très fortes intensités à l'extérieur et au large des deux baies (>0.5 m.s-1) et comprises entre 0.1 et $0.2 \mathrm{~m} . \mathrm{s}^{-1}$ à l'intérieur (figures $9 \mathrm{a}$ et $\mathrm{b}$ ). Lors de cette situation et au sud de la baie de Cintra, il a été observé qu'une partie de ces courants côtiers du large se réfractent à son cap situé au sud. Au milieu de la baie de Cintra et dans sa partie Nord, une partie des courants sont alors dirigés vers le Nord formant ainsi une circulation giratoire cyclonique impactant ainsi cette partie de la baie (Figure 9b). S'agissant de la baie de Dakhla, cette dernière ne serait pas impactée par ces courants du large (figure 9a). S'agissant de la baie de Dakhla et due à sa position géographique par rapport à l'orientation de ces courants du large, cette dernière n'en serait pas impactée lors des courants de jusant (figure 9a).
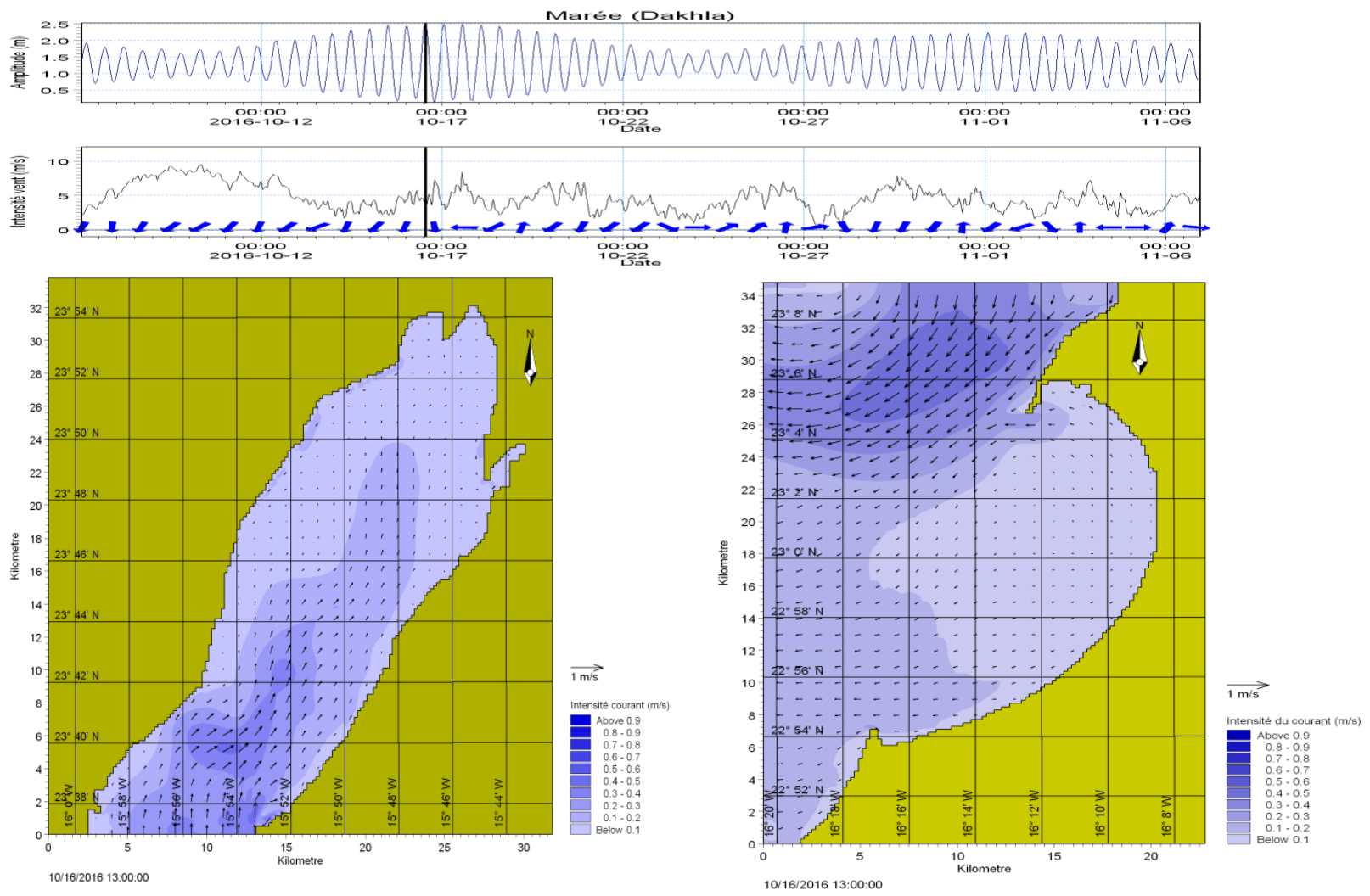

Figure 5:- Exemple de circulation des baies de Dakhla (à gauche) et Cintra (à droite) en période de marée de viveeau à marée entrante (cas du 16 octobre 2016 à $13 \mathrm{~h}$ - coefficient de marée 116). 

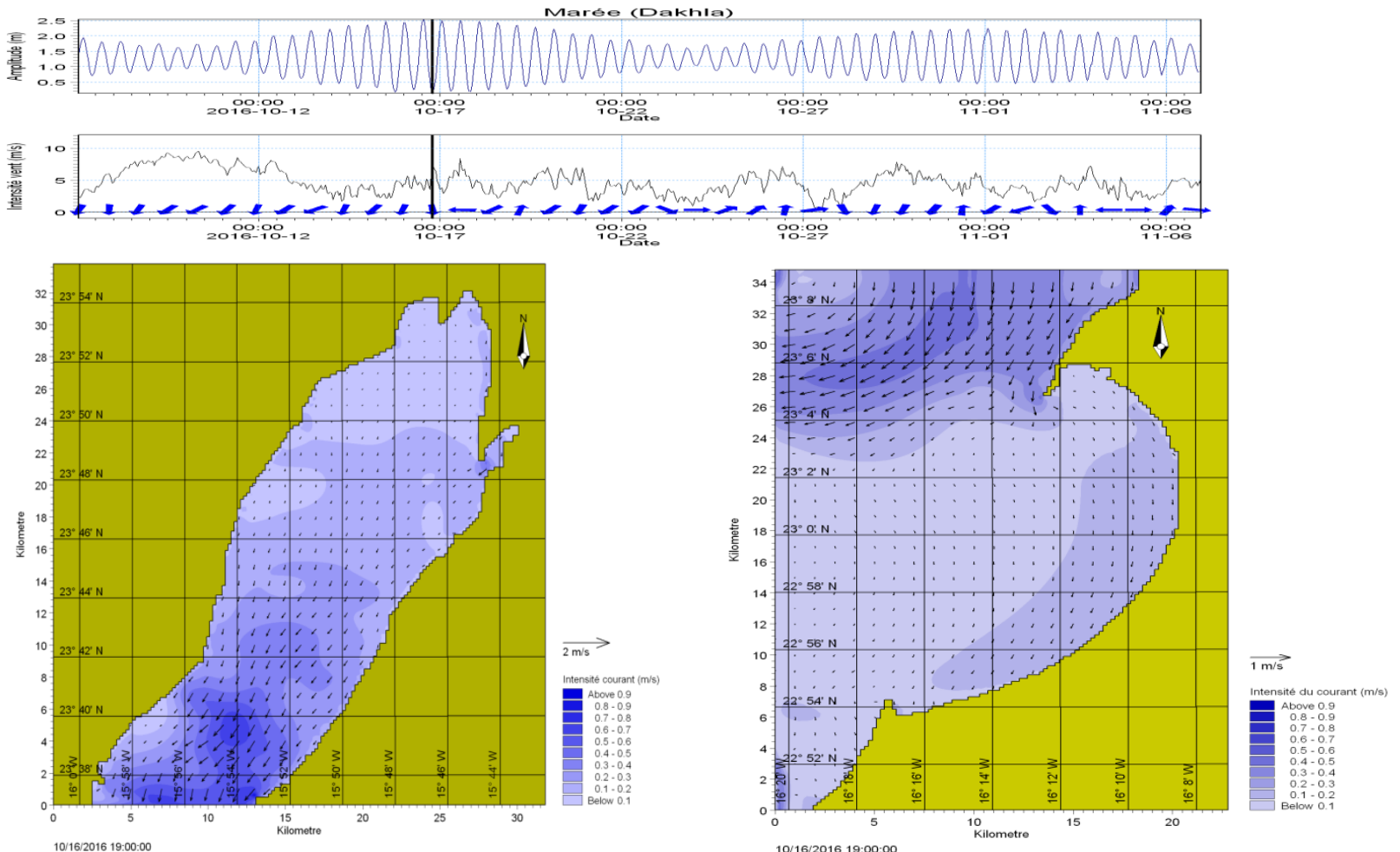

Figure 6:- Exemple de circulation des baies de Dakhla (à gauche) et Cintra (à droite) en période de marée de viveeau à marée sortante (cas du 16 octobre 2016 à 19h - coefficient de marée 116).
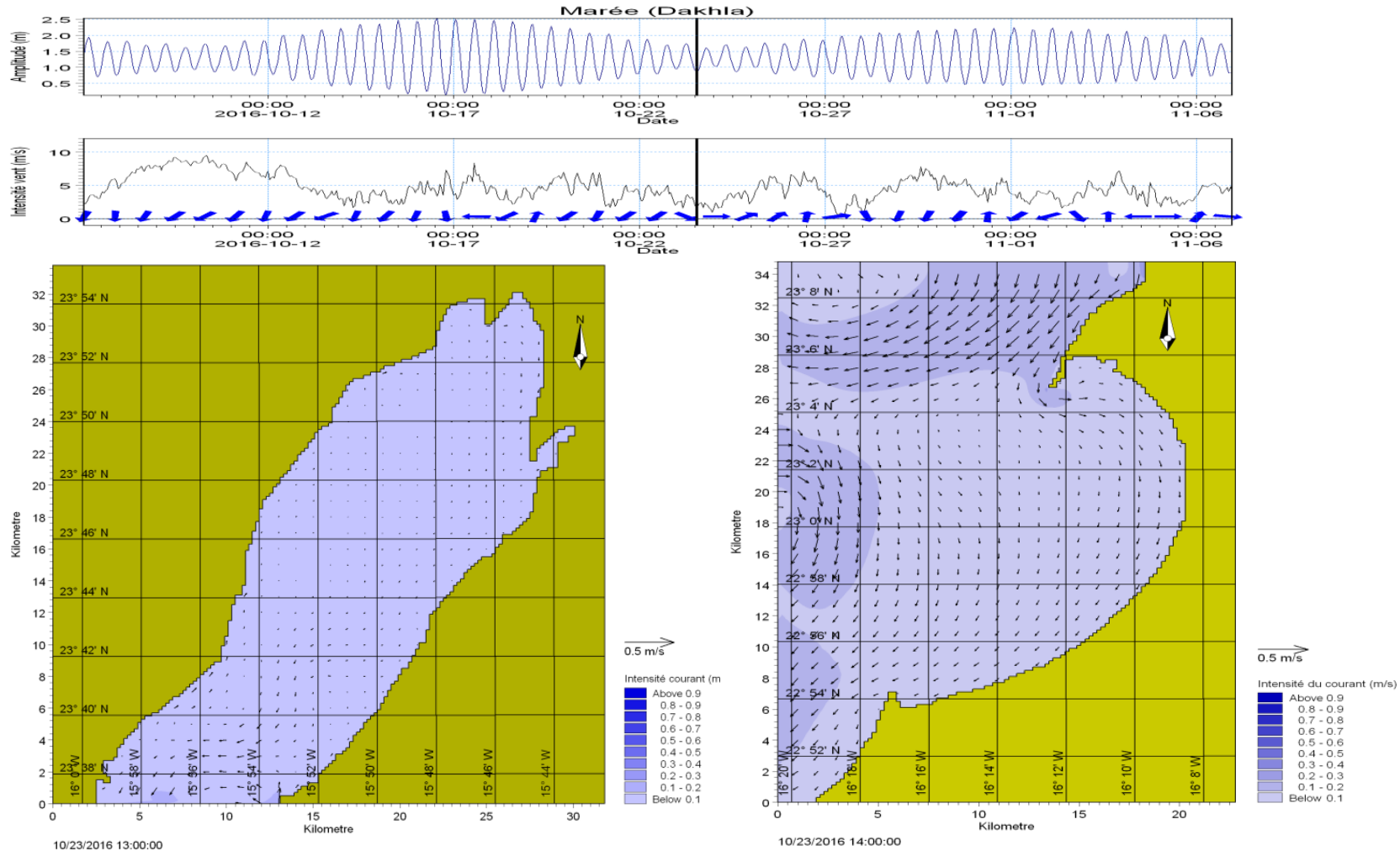

Figure 7:- Exemple de circulation des baies de Dakhla (à gauche) et Cintra (à droite) en période de marée de morte eau à marée sortante (cas du 23 octobre 2016 à 13h - coefficient de marée 31). 

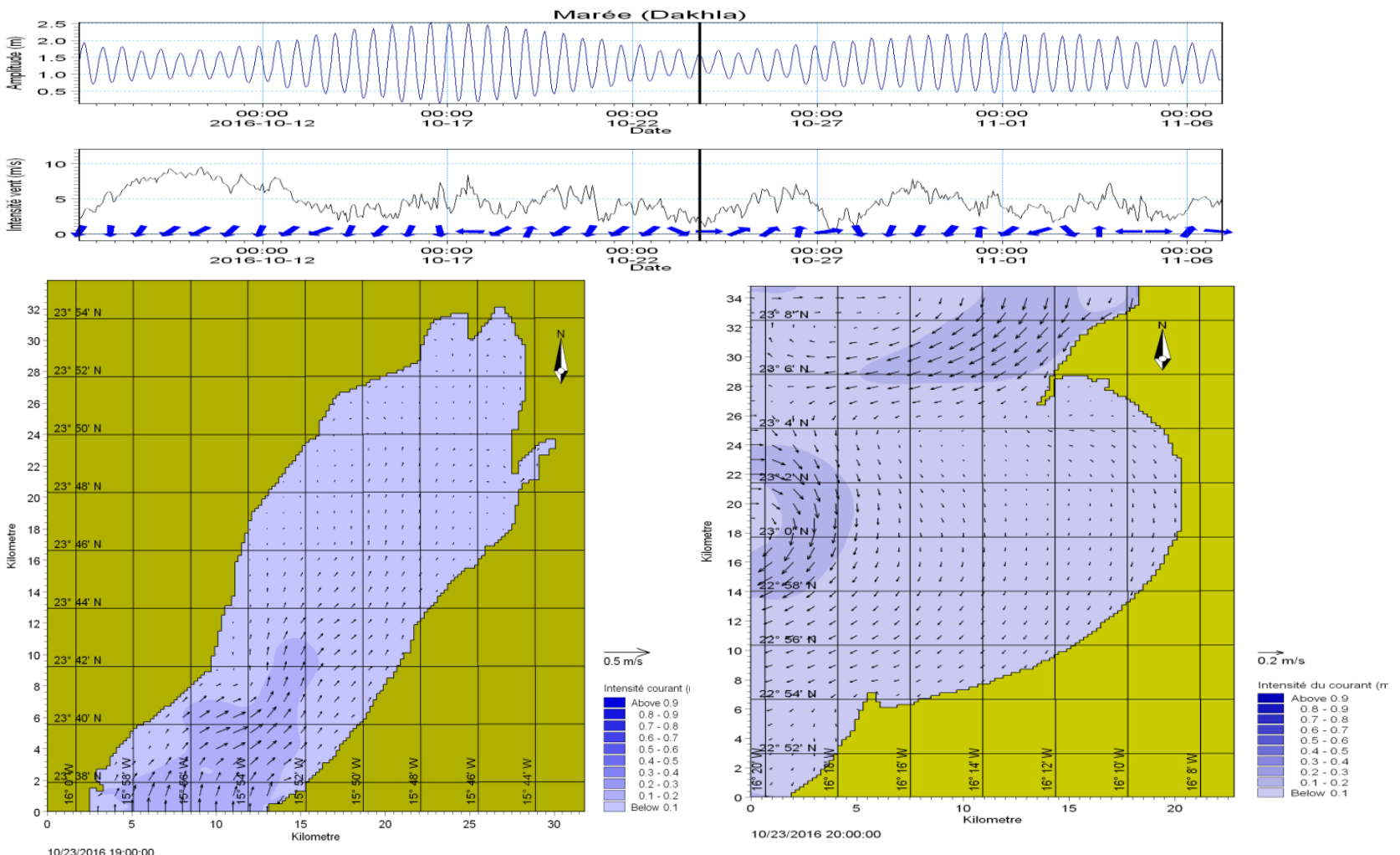

Figure 8:- Exemple de circulation des baies de Dakhla (à gauche) et Cintra (à droite) en période de marée de morte eau à marée entrante (cas du 23 octobre 2016 à 19h - coefficient de marée 31).
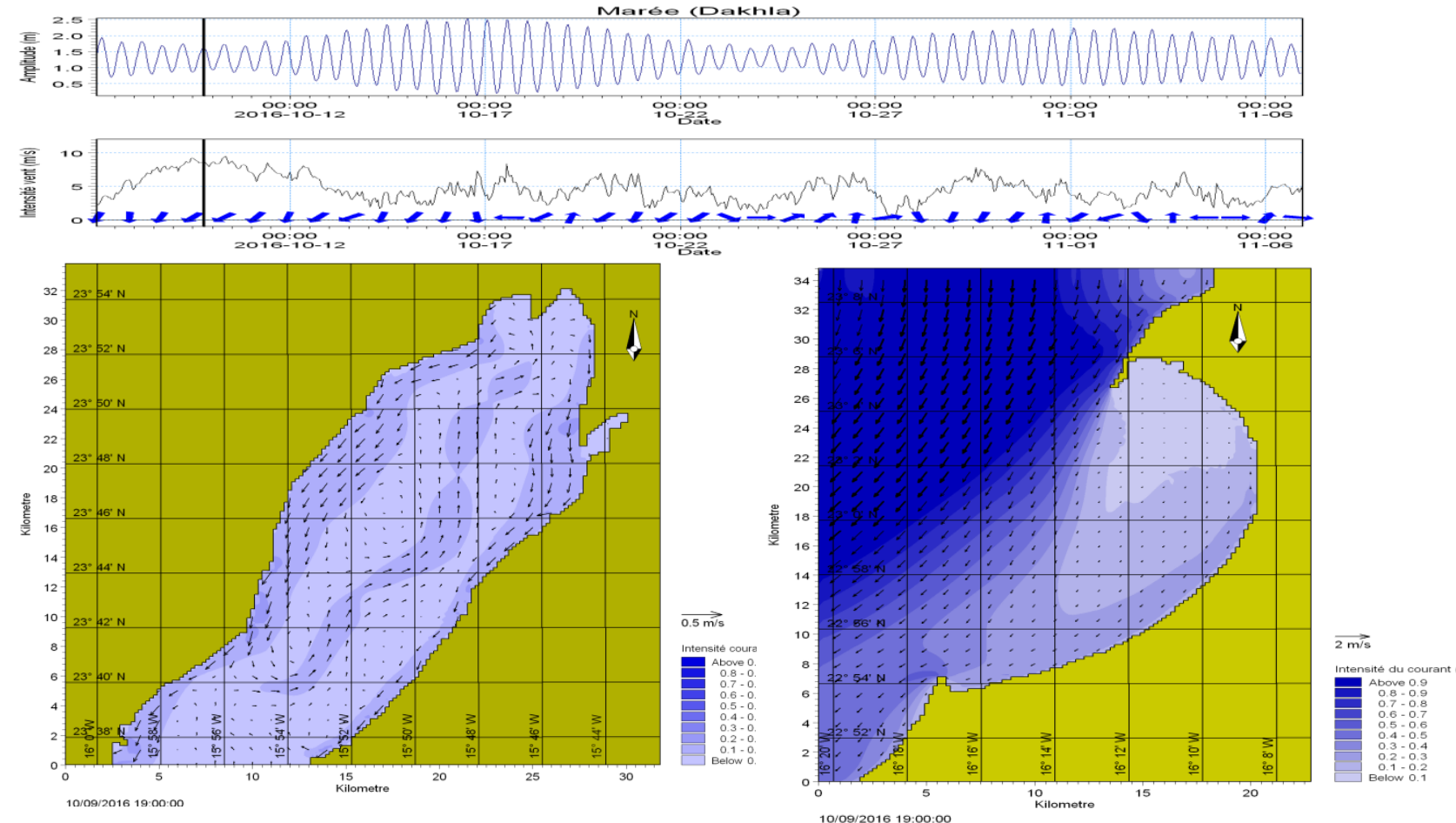

Figure 9:- Exemple de circulation des baies de Dakhla (à gauche) et Cintra (à droite) en période de marée de morte eau à marée entrante (cas du 09 octobre 2016 à 19h - coefficient de marée 41 et par vent Nord Est). A travers les différentes études de modélisation hydrodynamique menées au Maroc, c'est la baie de Dakhla qui présenterait de fortes intensités de courant observées lors des marées de vives eaux dont les intensités sont comprises 
entre 1 et 2 à la passe d'entrée et à son voisinage. Les courants de marée durant les jusants seraient légèrement supérieurs aux flots (Hilmi et al., 2017a). Au sein de la baie de Cintra, ces courants sont relativement plus faibles (< $0.3 \mathrm{~m} . \mathrm{s}^{-1}$ ) (Hilmi et al., 2017b). Comparativement à d'autres sites littoraux nationaux, les intensités des courants sont généralement comprises entre 1 et $1.2 \mathrm{~m} . \mathrm{s}^{-1}$ en périodes de marées de vives eaux. Ces forts courants ont été généralement observés au niveau des passes d'entrée des lagunes de Nador (Hilmi et al., 2005 et 2015; Koutitonsky et al., 2006; Marco et al., 2017; Umgeisser et al., 2005) et de Oualidia/Sidi Moussa (Hilmi et al., 2005; Koutistonky et al. , 2007 et 2012; Orbi et al., 2008;..). A l'intérieur de ces sites lagunaires, les courants sont généralement de moindres intensités, nettement inférieures à $0.5 \mathrm{~m} \cdot \mathrm{s}^{-1}$.

\section{Conclusion:-}

Cette étude a visé à étudier la circulation marine et le comportement hydrodynamique bidimensionnel des baies de Cintra et de Dakhla situées dans la région de Dakhla Oued Eddahab (Sud du Royaume du Maroc) soumises aux mêmes de conditions météorologiques et de marée allant du 07 octobre au 06 novembre 2016 . Soumises à l'influence des vents, les résultats de cette étude ont montré que les marées, principalement la marée semi diurne de type M2 qui est dominante sur la façade atlantique marocaine, dominent les courants de marée. Dans la baie de

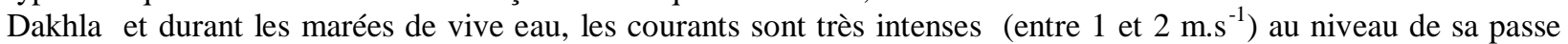
d'entrée et à son voisinage, inférieures à $1 \mathrm{~m} . \mathrm{s}^{-1}$ dans sa partie centrale de et inférieures à $0.5 \mathrm{~m} . \mathrm{s}^{-1}$ en se dirigeant vers l'amont de la baie. Durant les marées de mortes eaux, les intensités du courant de marée sont généralement moindres et inférieures à $0.4 \mathrm{~m} . \mathrm{s}^{-1}$ dans la baie de Dakhla. Dans la baie de Cintra, les courants présentent des intensités généralement supérieures à $0.4 \mathrm{~m} . \mathrm{s}^{-1}$ à l'extérieur de cette baie. Au sein de la baie, ces intensités sont inférieures à $0.3 \mathrm{~m} \cdot \mathrm{s}^{-1}$. D'autres travaux par modélisation hydrodynamique $3 \mathrm{D}$ des deux baies sont en cours de réalisation, notamment en vue d'estimer le temps de renouvellement de leurs eaux qui est un paramètre physique important à prendre en considération pour le développement des activités aquacoles dans ces baies.

\section{Remerciements:-}

Cet article est dédié à la mémoire de feu Dr Jaouad Lakhdar Idrissi, chercheur à l'Institut National de Recherche Halieutique qui a beaucoup travaillé de son vivant sur le site de la baie de Dakhla. Les auteurs tiennent aussi à remercier les équipes des Laboratoires Centraux de Casablanca et du Centre Régional de l'INRH à Dakhla qui ont contribué aux missions de terrain ainsi que les évaluateurs externes anonymes de la revue.

\section{References:-}

1. Abdellaoui, B., Nhhala, B., and Talbaoui, E.M. (2015). Mediterranean coastal lagoons: sustainable management and interactions among aquaculture, Capture fisheries and the environment: Studies and Reviews. General Fisheries Commission for the Mediterranean. № 95. Rome, FAO. p 163-173. ISBN 978-92-5-108792-3.

2. Alaoui, A. M., Choura, M., Maanan, M., Zourarah, B., Robin, M., Conceicao, M. F., Andrade, C., Khalid, M. and Carruseco, C. (2010). Metal fluxes to the sediments of the Moulay Bousselham lagoon, Morocco. Environmental Earth Sciences, July 2010, Volume 61, Issue 2, pp. 275-286.

3. ANDA (2015). Appel à Manifestation d'Intérêt - Plan de développement de l'aquaculture marine dans la région de Dakhla Oued Eddahab. Agence Nationale pour le Développement de l'Aquaculture, Royaume du Maroc, $24 \mathrm{p}$.

4. Arid, H., Moudni, H., Orbi, A., Talbaoui, E.M., Lakhdar, I.J., Massik, Z., Littaye, A. and Paoli, R. (2005). Télédétection spatiale et SIG pour la gestion intégrée des potentialités aquacoles. GEO OBSERVATEUR. Centre Royal de Télédétection Spatiale, $\mathrm{N}^{\circ}$ 14, pp 63-79.

5. Bajo, M., Abdellaoui, B., Maicu, F., Chair, A., Mesfioui, A., Layachi, M., El Madani, F., Hilmi, K. and Umgeisser, G. (2017). Changes in the water circulation of the Nador lagoon, due to anthropogenic modifications of the inlets. 19th EGU General Assembly (EGU2017), Proceedings from the conference held 2328 April, 2017 in Vienna, Austria., p.8185

6. Bocci, M., Brigolin, D., Pranovi, F., Najih, M., Nachite, D., and Pastres, R., (2016). An Ecosystem Approach for understanding status and changes of Nador lagoon (Morocco): application of food web models and ecosystem indices. Estuarine, Coastal and Shelf Science, Volume 171, pp 133-143.

7. Damsiri, Z., Elkalay, K, and Khalil, K. (2015). Modelling the biogeochemical cycle of the Oualidia lagoon (Atlantic, Morocco). International Journal of Advanced Research, Volume 3, Issue 2, 18-30, ISSN 2320-5407.

8. DHI (1990). Coastal Hydraulics and Oceanography. Mike 21. Hydrodynamic Module. User's Guide and reference Manual. 
9. El Khalidi, K., Zourarah, B., and Aajjane, A. (2011). Evolution récente de la morphologie de delta de flot et son effet sur la dynamique hydro-sédimentaire de la lagune de Oualidia (côte atlantique, Maroc): approche par photographie aérienne. Estudos do Quaternário, 7, APEQ, Braga, 2011, pp. 73-78.

10. Flower, R.J., Appleby, P.G., Thompson, J.R., Ahmed, M.H., Ramdani, M., Chouba, L., Rose, N., Orochester, R., Atyache, F., Kraiem, M., Elkhiati, N., El Kafrawy, S., Yang; H., and Rasmussen, E.K. (2009). Sediment distribution and accumulation in lagoons of the Southern Mediterranean Region (the MELMARINA Project) with special reference to environmental change and aquatic ecosystems. Hydrobiologia, Volume 622, Issue 1, pp 85-112.

11. Guelorget, O., Perthuisot, J.-P., Frisoni, G-F. and Monti, D. (1987). Le rôle du confinement dans l'organisation biogéologique de la lagune de Nador (Maroc). Oceanologica Acta, 10, 435-444.

12. Hamoumi, N. (2012). Le complexe lagunaire de Nador (Maroc): fonctionnement, contrôle naturel et provoqué, scenarii d'évolution future. Revue Paralia, Volume 5, pp 5.1-5.14.

13. Hassou, N., Maanan, M., Hennani, M., Zourarah, B., and Assobhei, 0. (2014). Spatial and temporal variation of faecal pollution indicators (Escherichia coli and faecal streptococci) and physico-chemical parameters at the Oualidia lagoon and its watershed (Morocco). International Journal of Current Microbiology and Applied Sciences, pp.675-694.

14. Hilmi, K., Orbi, A., Lakhdar, J.I. and Sarf, F. (2005a). Etude courantologique de la lagune de Oualidia (Maroc) en automne. Bulletin de l'Institut Scientifique, Section Sciences de la Vie, Rabat, $N^{\circ}$ 26-27, 67-71.

15. Hilmi, K., Koutitonsky, V.G., Orbi, A., Lakhdar, J.I., and Chagdali, M. (2005b). Oualidia Lagoon (Morocco): an estuary without a river. African Journal of Aquatic Sciences, Vol 30(1), 1-10.

16. Hilmi, K., Koutitonsky, V.G., Orbi, A., and Chagdali, M. (2005c) Three dimensional water circulation and dispersion in Nador lagoon (Morocco). In: P. Lasserre, P. Viarioli and P. Campostrini Eds: Lagoons and Coastal Wetlands, in the Global Change Context: Impacts and Management Issues,Proceedings of the International Conference, Venice, 26-28 April 2004, ICAM Dossier N³,UNESCO.

17. Hilmi, K., Orbi, A. and Lakhdar, J.I. (2009). Hydrodynamisme de la lagune de Oualidia (Maroc) durant l'été et l'automne 2005. Bulletin de l'Institut Scientifique, Section Sciences de la Terre, Rabat, N`31.

18. Hilmi, K., Orbi, A., Makaoui, A., Bouksim, H., Idrissi, M., Ettahiri, O., Benyounes, A., and Ait Chattou, M. (2017a). Circulation marine de la baie de Dakhla (Sud du Maroc) par modèle hydrodynamique 2D. European Scientific Journal, March 2017 Edition vol.13, No.9, pp 69-82, ISSN: 1857 - 7881 (Print).

19. Hilmi, K., Makaoui, A., Ettahiri, O., Idrissi, M., Abdellaoui, B., Agouzouk, A., BaiBai, T., Ait Chattou, M. , Bessa I., and Bouksim, H. (2017b.) Circulation marine de la baie de Cintra (Sud du Maroc) par modèle hydrodynamique 2D. European Scientific Journal April 2017 Edition vol.13, No.12 ISSN: 1857 - 7881 (Print) e - ISSN 1857- 7431, 175-194.

20. Idardare, Z., Moukrim, A., Chiffoleau, J.-F., Ait Alla, A., Auger, D. and Rozuel, E. (2013). Evaluation de la contamination métallique dans deux lagunes marocaines: Khnifiss et Oualidia, Revue Marocaine des Sciences Agronomiques et Vétérinaires, Vol. 1, No 2 (2013).

21. Koutitonsky, V.G, Orbi, A., Hilmi, K., and Berraho, Ab. (2006). Benefits of a proposed second inlet on water renewal in Nador lagoon, Morocco. The 1st International Conference: Environmental Change in lakes, lagoons and wetlands of the southern Mediterranean region, 4-7 january 2006, Cairo, Egypt.

22. Koutitonsky, V.G., Ouabi, M. and Ibrahimi. I. (2007). L'étude du comportement hydrosédimentaire du système lagunaire Oualidia par la modélisation mathématique. Phase 2 : Modélisation hydro-sédimentaire de l'état actuel et de scenarios d'aménagement. Direction des Ports et du Domaine Public Maritime, Ministère de l'Équipement et du Transport, Royaume du Maroc. 204 p + Annexes .

23. Koutitonsky, V. G., Zyserman, J., and Zourarah, B. (2012). Étude par modèle mathématique de l'impact de l'ouverture de la digue amont et de l'enlèvement ou redistribution des sédiments de la sablière sur le comportement hydrosédimentaire de la lagune d'Oualidia. Mission 2 : Modélisation hydro-sédimentaire de l'état actuel et des scénarios d'aménagement. Direction des Ports et du Domaine Publique Maritime, Ministère de l'Équipement et du Transport, Royaume du Maroc, 303 p. + Annexes.

24. Labbardi, H, Ettahiri, O., Lazar, S., Massik, Z., and El Antri, S. (2005). Étude de la variation spatio-temporelle des paramètres physico-chimiques caractérisant la qualité des eaux d'une lagune côtière et ses zonations écologiques: cas de Moulay Bousselham, Maroc. Comptes Rendus Geoscience, Vol. 337, Issue 5, pp 505-514.

25. Lakhdar, J.I., Orbi, A., Hilmi, K. and Zidane, F. (2001). Etude hydrochimique et courantologique d'un milieu paralique ; la lagune de Khnifiss (Sud du Maroc). L'Eau, L'Industrie et les Nuisances, N 250, pp.24-28.

26. Lakhdar, J.I., Orbi, A, Hilmi, K., Zidane, F., Sarf, F., Massik, Z and Makaoui, A., (2004). Organisation et fonctionnement d'un écosystème côtier du maroc: la lagune de Khnifiss. Revue Sciences de l'Eau, 17(4), pp 447-462. 
27. Lakhdar, J.I., Orbi, A., Hilmi, K., Zidane, F. and Moncef, M. (2005). Modèles de température et de la qualité de l'eau appliqués à deux lagunes marocaines : Oualidia (Atlantqiue) et Nador (Méditerranée). Environmental Technology, Vol. 26, 713-720..

28. Maanan, M., Ruiz-Fernandez, A.C., Maanan, M., Fattal, P. and Zourarah, B. (2014). A long-term record of land use change impacts on sediments in Oualidia lagoon, Morocco. International Journal of Sediment Research, 29 (1), 1-10.

29. Maanan, M., Saddik, M., Maanan, M., Chaibi, M., Assobhei O. and Zourarah, B. (2015). Environmental and ecological risk assessment of heavy metals in sediments of Nador lagoon, Morocco. Ecological Indicators, Vol. 48, pp 616-626.

30. Mostarih, M.M.M., El Madani, F., Yahya, H.S.A, El Hachemi, O. , Abdellaoui, S. and Chafi, A. (2016). Evaluation physico-chimique de la qualité de l'eau de la lagune de Nador - Nord du Maroc oriental - après l'ouverture de la nouvelle passe. J. Mater. Environ. Sci. , 7 (12), 4795-5809 ISSN : 2028-2508 CODEN: JMESCN.

31. Najih, M., Berday, N., Rezzoum, N., Lamrini, M.F., and Nachit, A. (2016). Evolution temporelle et spatiale de la macroflore benthique de l'écosystème lagunaire de Nador. European Scientific Journal, December 2016 Edition vol.12, No.36, pp 355-377, ISSN: 1857 - 7881 (Print).

32. Orbi, A., Hilmi, K., Lakhdar, J.I., and Zizah, S. (2008). Lagoon Ecosystem Study Trough Two Cases: Oualidia (Atlantic) and Nador (Mediterranean) - Morocco. Chapter In: Sustainable Use and Development of Watersheds - Part of the series NATO Science for Peace and Security Series pp 289-298.

33. Saad, Z., Orbi, A., Abouabdellah, R., Saad, A., and Oudra, B. (2013). Impact of economic development on the dynamics of phytoplankton and physic-chemical quality of Dakhla Bay (South of Morocco). South Asian Journal of Experimental Biology, 3 (5), 274-285.

34. Saad, Z., El-Gharmali., A., El-Khalloufic, F., Saad., A., and Oudra, B. (2015). Metal contamination of the macrobenthic fauna of the Dakhla Bay (South of Morocco): an ecotoxicological diagnostic and environmental perspective. South Asian Journal of Experimental Biology, Vol.5 No.2 pp.55-69.

35. Umgiesser, G., Chao, J., Bajo, M., Scroccaro, I., and Cucco, A. (2005). Residence time modelling in the Nador lagoon, Morocco, In Proceedings of the First International Conference on Coastal Conservation and Management in the Atlantic and Mediterranean (ICCCM'05), F. Veloso Gomes, F. Traveira Pinto, L. das Neves, A. Sena and O. Ferreira (eds), pp. 389-397, 2005.

36. Umgiesser, G., Ferrarin, C., Cucco, A., De Pascalis, F., Bellafiore, D., Ghezzo, M. and Bajo, M. (2014). Comparative hydrodynamics of 10 Mediterranean lagoons by means of numerical modeling, J. Geophys. Res. Oceans, 119, 2212-2226, doi:10.1002/2013JC009512.

37. Yachouti, A., Daoudi, S., ElMahi I., and Boushaba, F. (2014). A Numerical Model for the Simulation of Water Recirculations in the Nador Lagoon (Morocco). International Journal of Hydraulic Engineering, 3(4): 111-119 DOI: $10.5923 /$ j.ijhe.20140304.02.

38. Zidane, H., Orbi, A., Mouradi, A., Zidane, F. and Blais, J-F. (2008). Structure hydrologique et édaphique d'un site ostreicole: Duna Blanca (la baie de Dakhla sud du Maroc). Environnemental Technology, Vol. 29, pp 10311042.

39. Zidane, H. (2011). Potentialités aquacoles de la baie de Dakhla. Environnemental Technology, Vol. 29, pp 1031-1042.

40. Zidane, H., Menioui, M., Mouradi, A., Orbi, A. and Zidane, F (2013a). Croissance de l'huître C. Gigas, en élevage dans la baie de Dakhla - Maroc, Rapp. Comm. int. Mer Médit., CIESM 40. p. 470.

41. Zidane, H., Menioui, M., Mouradi, A., Orbi, A., Zidane, F. and Blais,J-F. (2013b). Les métaux lourds dans le sédiment et leur impact sur le développement de la conchyliculture (Baie de Dakhla, Maroc). Rapp. Comm. int. Mer Médit., CIESM 40, p. 766.

42. Zourarah, B., Aajjane, A., El khalidi, K., Koutitonsky, V.G., and Zyserman, J. (2012). Étude par modèle mathématique de l'impact de l'ouverture de la digue amont et de l'enlèvement ou redistribution des sédiments de la sablière sur le comportement hydrosédimentaire de la lagune de Oualidia. Mission 1 : Etude préliminaire. Direction des Ports et du Domaine Public Maritime, Ministère de l'Équipement et du Transport, Royaume du Maroc, 96 p. + Annexes. 